\title{
A REPRESENTATION THEOREM FOR ARCHIMEDEAN LINEAR LATTICES ${ }^{1}$
}

\section{LEON BROWN AND HIDEGORO NAKANO}

A function lattice $(f v g=\max (f, g))$, as well as linear (vector) lattices of functions are Archimedean. In this note we present a characterization of Archimedean linear lattices as quotient spaces of function lattices.

The general problem was brought to our attention when Professor Paul C. Shields asked us whether there is an example which cannot be represented by any quotient space of function lattices. Note that $L_{p}(p \geqq 1)$ of a nonatomic measure space cannot be represented as a function lattice [7], but they are quotient spaces of function lattices.

Let $S$ be a linear lattice. A subset $N$ is called an ideal whenever $N$ is a linear subspace of $S$ and $|x| \leqq|y|, y \in N$ implies $x \in N$. An ideal $N \subset S$ is called $\sigma$-normal whenever $0 \leqq u_{n} \in N(n=1,2, \cdots)$ and $u_{n} \uparrow u$ ( $u_{n}$ is increasing and $\sup u_{n}=u$ exists) implies $u \in N . S$ is

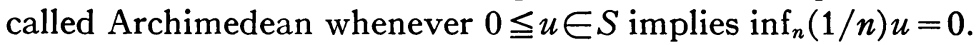

$S / N$ is a linear lattice if and only if $N$ is an ideal in $S[2$, p. 222 and p. 238]. The following example shows that even though $S$ is Archimedean, $S / N$ need not be.

ExAmple. Let $S$ be the space of all sequences and $N$ be all null sequences. If $i(n)=n$ and $e(n)=1$ for $n=1,2, \cdots$, then $(1 / k)(i+N)$ $\leqq e+N$ for all $k$ and thus $S / N$ is not Archimedean.

The following theorem gives us a necessary and sufficient condition for $S / N$ to be Archimedean. It was suggested to us by the referee and provides a generalization of Corollary 1 , as well as a simpler proof. We wish to thank the referee for his helpful suggestions.

Theorem 1. Let $S$ be a linear lattice and let $N$ be an ideal in $S$. Then $S / N$ is Archimedean if and only if $N$ has the following property: $0 \leqq u$, $v \in S$ and $(v-(1 / n) u)^{+} \in N(n=1,2, \cdots)$ implies $v \in N$.

Proof. Let $u+N, v+N \in S / N$ and assume $0 \leqq v+N \leqq(1 / n)(u+N)$ for $n=1,2, \cdots$. Let $0 \leqq u$, then for every $n(n=1,2, \cdots)$ there is an element $v_{n} \in v+N$ such that $v_{n} \leqq(1 / n) u$. Since $v_{1} \wedge v_{n} \in v+N$ for all $n$, we may assume $v_{n} \leqq v_{1}$ and $v_{1} \geqq 0$. Now, $N \ni v_{1}-v_{n} \geqq\left(v_{1}-(1 / n) u\right)^{+}$ for $n=1,2, \cdots$ which implies by hypothesis that $v_{1} \in N$ and so $v+N=0$ proving $S / N$ is Archimedean.

Presented to the Society, April 12, 1965; received by the editors August 17, 1965.

1 This research was supported in part by National Science Foundation Grant No. GP-54. 
To prove the converse, suppose $0 \leqq u, v \in S$ and $(v-(1 / n) u)^{+}$ $\in N(n=1,2, \cdots)$. Then $(1 / n) u+(v-(1 / n) u)^{+} \geqq v$ which implies that $(1 / n)(u+N) \geqq v+N$ for $n=1,2, \cdots$, and hence $S / N$ Archimedean implies $v \in N$.

q.e.d.

If $S$ is Archimedean, then $(v-(1 / n) u)^{+} \uparrow v(0 \leqq u, v)$ and we have the following result:

Corollary 1. If $S$ is Archimedean and if $N$ is a $\sigma$-normal ideal, then $S / N$ is an Archimedean linear lattice.

Another example of Theorem 1 is the following: If $0 \leqq \phi$ is a nonnegative linear functional on $S$, then the null-ideal $N_{\phi}=\{x \mid \phi(|x|)$ $=0\}$ satisfies the condition of the theorem. Indeed, $(v-(1 / n) u)^{+}$ $\in N_{\phi}$ implies that $\phi(v) \geqq \phi\left((v-(1 / n) u)^{+}\right)=0 \geqq \phi(v)-(1 / n) \phi(u)$ $(n=1,2, \cdots)$, and so $\phi(v)=0$.

Let $\bar{R}$ denote the two-point compactification of the real line $R$ and let $X$ be a topological space. A mapping $f$ of $X$ in to $\bar{R}$ will be called an extended function whenever $f$ is continuous and the open set $R(f)$ $=\{x \mid f(x) \in R\}$ is everywhere dense in $X$. Let $D(X)$ denote the set of all extended-valued functions. If $f, g \in D(X)$ and $a$ is real, then the functions $a f, \max (f, g)$ and $\min (f, g)$ which are defined pointwise are in $D(X)$. If there is a $h \in D(X)$ such that $h(x)=f(x)+g(x)$ for all $x \in R(f) \cap R(g)$, then $h$ is called the sum of $f$ and $g$. Since $R(f) \cap R(g)$ is dense in $X$, the sum is uniquely defined if it exists. A subset $L$ of $D(X)$ which is a linear space with respect to these four operations is called a linear space of extended functions on $X$.

Any Archimedean linear lattice $S$ can be embedded in the Dedekind completion of $S$ [6, pp. 140-147 (cut extension)] which is a complete linear lattice. This fact and the following theorem proved for $\sigma$ complete linear lattices [8] gives us

THEOREM 2. Every Archimedean linear lattice is linear and lattice isomorphic to a linear lattice of extended functions on a locally compact Hausdorff space.

We note that representation theorems in terms of continuous extended real-valued functions on some Hausdorff space have been constructed by Yoshida [11], Maeda and Ogasawara [4], Amemiya [1], Vulih [10] and more recently by Papert [9], Johnson and Kist [3], as well as by Nakano [8]. Also Johnson and Kist prove that the topological space constructed by Yoshida is locally compact.

Using Theorem 2, we are able to prove the following representation theorem. 
TheOREM 3. If $S$ is an Archimedean linear lattice, then there exists a lunction lattice $F$ and a $\sigma$-normal ideal $N \subset F$ such that $S$ is linear and attice isomorphic to $F / N$.

Proof. Let $S$ be an Archimedean linear lattice and let $L=L(X)$ be the linear lattice of extended functions on the locally compact Hausdorff space which is linear and lattice isomorphic to $S$. If $f, g$ $\in L(X)$ and $\{x \mid f(x) \neq g(x)\}$ is of the first category then $f=g$. This follows from the fact that since $X$ is locally compact, $X$ is of second category, and so the complement of a set of the first category is dense in $X$.

We define the set $\mathfrak{L}=\mathfrak{L}(X)$ of real functions on $X$ as follows: $f \in \mathfrak{L}$ if there exists an element $h_{f} \in L$ such that $\left\{x \mid f(x) \neq h_{f}(x)\right\}$ is of first category. From the observation made above it follows that $h_{f}$ is uniquely determined. Let $T$ denote the mapping $f \rightarrow h_{f}$ of $\mathscr{L}$ onto $L$. Then one easily sees that $\mathscr{L}$ is a linear lattice of real functions on $X$ and that the transformation $T$ has the following properties: (i) $T$ is linear and (ii) $T(\max (f, g))=\max (T f, T g)$. Finally, let $N=\{f \mid f \in \mathcal{L}$ and $T f=0\}$. Then $f \in N$ if and only if $f=0$ except on a set of first category, and so $N$ is a $\sigma$-normal ideal in $\mathscr{L}$ such that $\mathscr{L} / N$ and $L$ are linear and lattice isomorphic. This completes the proof.

Observe that Corollary 1 implies that since $L$ is Archimedean then $\mathscr{L} / N$ is Archimedean.

\section{REFERENCES}

1. I. Amemiya, A general spectral theory in semi-ordered linear spaces, J. Fac. Sci. Hokkaido Univ. Ser. I 12 (1953), 111-156.

2. G. Birkhoff, Lattice theory, Amer. Math. Soc. Colloq. Publ., Vol. 25, Amer. Math. Soc., Providence, R. I., 1948.

3. D. G. Johnson and J. E. Kist, Prime ideals in vector lattices, Canad. J. Math. 14 (1962), 517-528.

4. F. Maeda and T. Ogasawara, Representation of vector lattices, J. Sci. Hiroshima Univ. Ser. A 12 (1942), 17-35. (Japanese)

5. H. Nakano, Modern spectral theory, Maruzen, Tokyo, 1950.

6. —- Modulared semi-ordered linear spaces, Maruzen, Tokyo, 1950.

7. —— Über ein lineares Funktional auf dem teilweise geordneten Modul. Proc. Imp. Acad. Tokyo 18 (1942), 548-552.

8. - Eine spectraltheorie, Proc. Phys.-Math. Soc. Japan 23 (1941), 485-511.

9. D. Papert, $A$ representation theory for lattice-groups, Proc. London Math. Soc. (3) 12 (1962), 100-120.

10. B. Vulih, Some questions of the theory of linear partially ordered sets, Izv. Akad. Nauk. SSSR Ser. Mat. 17 (1953), 365-380.

11. K. Yoshida, On the representation of the vector lattice, Proc. Imp. Acad. Tokyo 18 (1942), 339-342.

Wayne State University 